\title{
Abnormal Meiosis in Grain Sorghums-Desynapsis
}

\author{
N. Krishnaswamy and Miss K. Meenakshi' \\ Cytogenetics Laboratory, Agricultural Research Institute, \\ Coimbatore, India
}

Received February 19, 1957

Studies on meiotic abnormalities have always been interesting and useful. Asynapsis has been attributed to several causes such as temperature (Pao \& Li. 1948), age, failure of chromosomal spiralization etc. $\mathrm{Li}$ et al (1945) suggested the restriction of the term 'desynapsis' to cases where some amount of prophasic pairing takes place and later the homologues separate out so that at diakinesis most of them are univalents. The majority of cases investigated under this type of abnormality viz., asynapsis, have been found to be desynaptic and actually very few are asynaptic sensu stricto. Both kinds are found to be gene controlled and usually behave as simple Mendelian recessives.

Huskins and Smith (1934) reported the occurrence of asynapsis in sorghum and considered this as due to premature splitting and irregular contraction. In the present report have been described two types of desynapsis in grain sorghums.

\section{Materials and methods}

These two types were first met with in segregating populations. The first (A. S. 8486/1) was noted in an F.-6., progeny of a cross between $S$. durra var. Coimbatoricum Snowden (A.S. 60) X S. caudatum (A.S. 4003, Bonganhilo, an African sorghum) X A.S. 60 in the year 1953. The desynaptic plants were transplanted into an irrigated field and kept growing. The second type is from a pure line (Sen Cholam, Punjaipuliampatti, A.S. 681) of S. subglabrescens. Here also the sterile plants were transplanted into an irrigated field and kept growing. (The two types have been designated as Dsy-1 and Dsy-2).

The materials for meiotic study were collected mainly from the ratoons of the transplanted sterile plants. The flowers were fixed in acetic alcohol 1:3 mixture. Both temporary acetocarmine preparations and permanent smears stained in gentian-violet-iodine and also in Feulgen nucleal reaction were used for study. All figures are drawn to a magnification of $\times 2100$ in the case of Dsy-1 and $\times 2300$ in Dsy-2, unless otherwise mentioned. Microphotographs were taken with a Zeiss Ikon Kolibri camera and enlarged.

1 Assistant in Millets Breeding Section. 
All figures and microphotographs were reduced to half in reproduction.

\section{Observations}

A.S. 60 and A.S. 4003 are both season bound i.e. they come to flower only during the cold season, November-December. The lemmas close some time after pollination and the stigmas soon fade in the fertile plants. The vegetative growth in the two sterile plants was in no way different from the fertile ones. The plant Dsy-1 on ratooning grew up vigorously especially towards the flowering season. These, however, flowered of tener than the parents and irrespective of season. The stems grew into thick, canes about $450 \mathrm{~cm}$ long and when allowed to lie on the ground struck roots at nodes and shoots also were produced. The panicles are semicompact and normal in appearance. The exsertion of anthers and stigma is normal. The former do not dehisce and are mostly shrivelled. The stigmas are viscid and fully exserted. The lemmas keep open for quite a long period and similarly the stigmas keep fresh especially when the panicles are enclosed in a butter paper bag. On selfing rarely any seeds set.

In the second type (Dsy-2) the plants are also vigorous and characters are similar to the normal ones. As in the first type the flowers keep open and fresh for a longer time than in normal heads. The anthers shrivel and rarely dehisce. On selfing a few seeds set. Here also the ratoon came to flower within two months of transplanting.

Meiosis. Dsy-1 : Late pachytene showed normal behaviour of the paired and unpaired chromosomes. At diakinesis the chromosomes become shortened and thickened, (Fig. 1, full desynapsis having $20_{\mathrm{I}}$; Fig. 2a, $4_{\mathrm{II}}+12_{\mathrm{I}}$; Fig. $2 \mathrm{~b}$, full synapsis with $10_{\text {II }}$ ). Fig. $2 \mathrm{c}$ shows a P.M.C at diakinesis with $10_{\mathrm{I}}$ but the cell has already developed thickened wall and in Fig. 2d is shown a rare occurrence of a probable syncyte-like cell at diakinesis. The nucleolar chromosomes are sometimes paired and sometimes remain single. There are two pairs of nucleolar chromosomes. The number of bivalents formed varies from $0-10$. Table 1 gives the frequencies.

The metaphase is normal with a bipolar spindle and chromosomes well congressed (Fig. 3). Rarely univalents are found away from the equatorial plate. Anaphase is normal except in rare cases. The bivalents undergo reduction but the unpaired chromosomes are distributed at random. Frequently equal distribution of chromosomes is found giving 10-10 in each daughter cell (Fig. 4). Rarely spindle may show lack of coalescing (Fig. 5), but this does not appear to affect the cytokinesis or lead to the formation of multiple nuclei. Fig. 6 shows an abnormal P.M.C. with thickened wall and 10-12 distribution of the chromosomes. Daughter cells of unequal sizes are also found.

Usually telophase is normal with interkinetic wall formation and com. plete migration of all the chromosomes into the daughter nuclei (Fig. 7 with 
10-10 distribution). Normal interkinesis is found (Fig. 8).

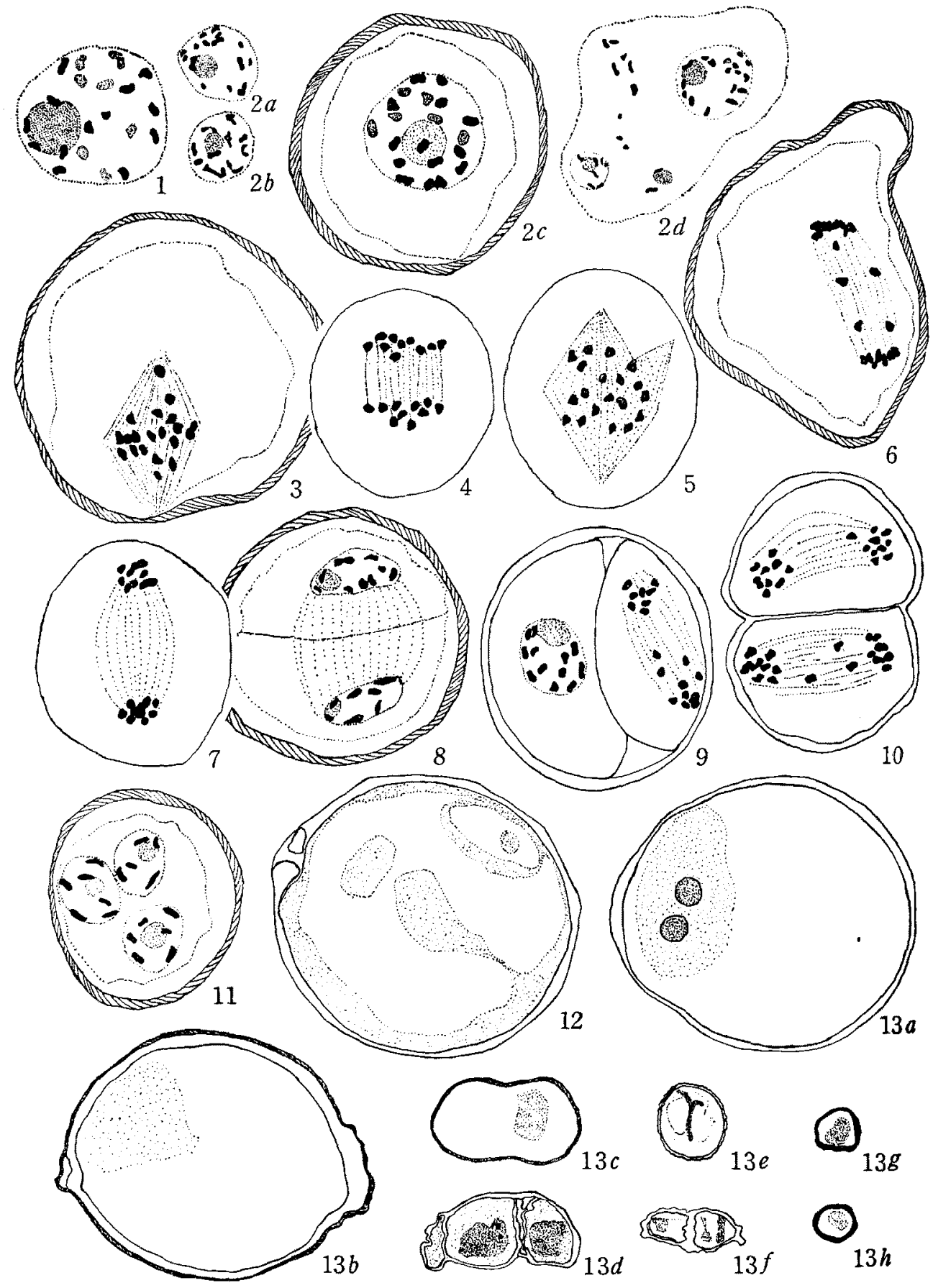

Figs. 1-13. Explanations are given in text. 1-13h, Meiosis in Dsy-I. 1-12, $\times 1050.13 a \sim h$, $x$ ca 760 .

Second division again shows both equational and random distribution of chromosomes thereby giving rise to differences in the chromosome numbers 
of the daughter cells. Fig. 9 shows a second division P.M.C. in which there has been 9-8 distribution of undivided chromosomes and three which have undergone equational division giving at telophase-I a total of 12-11 distribution and in anaphase-II in one daughter cell, the three chromosomes which had undergone equational division do not divide but migrate to one pole while the others undergo equational division giving ultimately 8-11 distribution. Frequently 10-10 distribution is found (Fig. 10). Tetrad division is gone through and normal looking rounded pollen grains are formed. Fig. 11 represents a rare case of a triad formation. Size differences are seen in the tetrad cells. At maturity almost all pollen grains are empty. Fig. 12 shows a giant pollen grain gradually disorganising. Very rarely a few normal ones with contents are found. Fig. $13 \mathrm{a}$-h show the abnormal pollen representing the size variations and also in different stages of disorganisation ultimately becoming empty. The size frequency of the pollen grains taken as diameters is given in Table 3.

Table 1. Number of bivalents formed

\begin{tabular}{|c|c|c|c|c|c|c|c|c|c|c|c|c|c|}
\hline \multirow{2}{*}{ Plant } & \multicolumn{13}{|c|}{ Number of P.M.C. with bivalents } \\
\hline & 0 & 1 & 2 & 3 & 4 & 5 & 6 & 7 & 8 & 9 & 10 & Total P.M.C. & Mean/P.M.C. \\
\hline Dsy-1 & 8 & 4 & 2 & 2 & 4 & 1 & 5 & 1 & 3 & 4 & 31 & 65 & 6.5 \\
\hline Dsy-2 & 31 & 9 & 3 & 1 & - & - & - & - & - & - & 1 & 45 & 0.62 \\
\hline
\end{tabular}

Dsy-2: At late diplotene the chromosomes are found long and show coiling as in somatic chromosomes but there is no conjugation. There was also no indication of homologous chromosomes lying near by as in somatic pairing or distance conjugation. Diakinesis shows all chromosomes condensed, much reduced and towards late diakinesis almost spherical. However, the P.M. cells have only univalents (Fig. 14 a) and rarely one bivalent. Very rarely cells with full synapsis and bivalent formation are met with. The frequencies of bivalent formation are given in Table 1. Fig. $14 \mathrm{~b}$ shows an abnormal P.M.C. showing two chromosomes forming separate nucleus.

The metaphase (Figs. 15 and 16 , early $\mathrm{M}-\mathrm{I}$ with persistent nucleolus) shows a well formed bipolar spindle (Fig. 17 a) and full congression at the equatorial plate. The plate is compact as in normal meiosis but it appears to be wider than in P.M.Cs with normal synapsis. The chromosomes are also considerably reduced and condensed as though conjugation had taken place. The nucleolus is seen to persist beyond diakinesis. Fig. $17 \mathrm{~b}$, is a polar view of metaphase-I showing persistent nucleolus.

Anaphase is irregular owing to the random distribution of the univalents. The bivalents invariably undergo reduction division. Usually the majority of the univalents migrate without division but equational division of a few of the univalents also occurs. In Fig. 18 is shown a P.M.C. with 12-8 distribution and a persistent nucleolus. Some of the univalents may lag 
behind the others, though this is not usual. (Fig. 19 shows 6-9 distribution of undivided univalents, four at the equatorial plate and one having undergone equational division giving $6+1-4-9+1$.) Usually all the
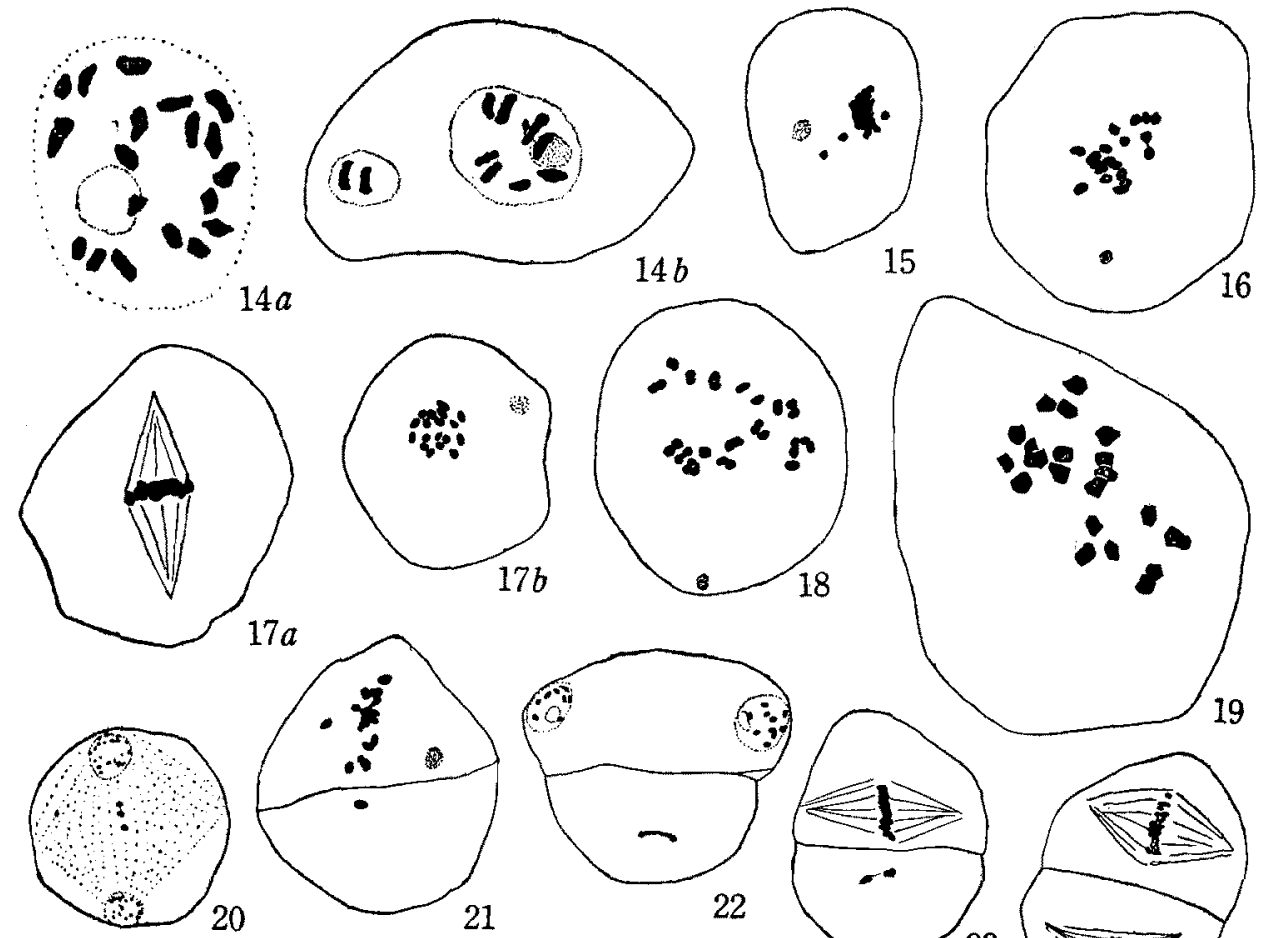

21

22
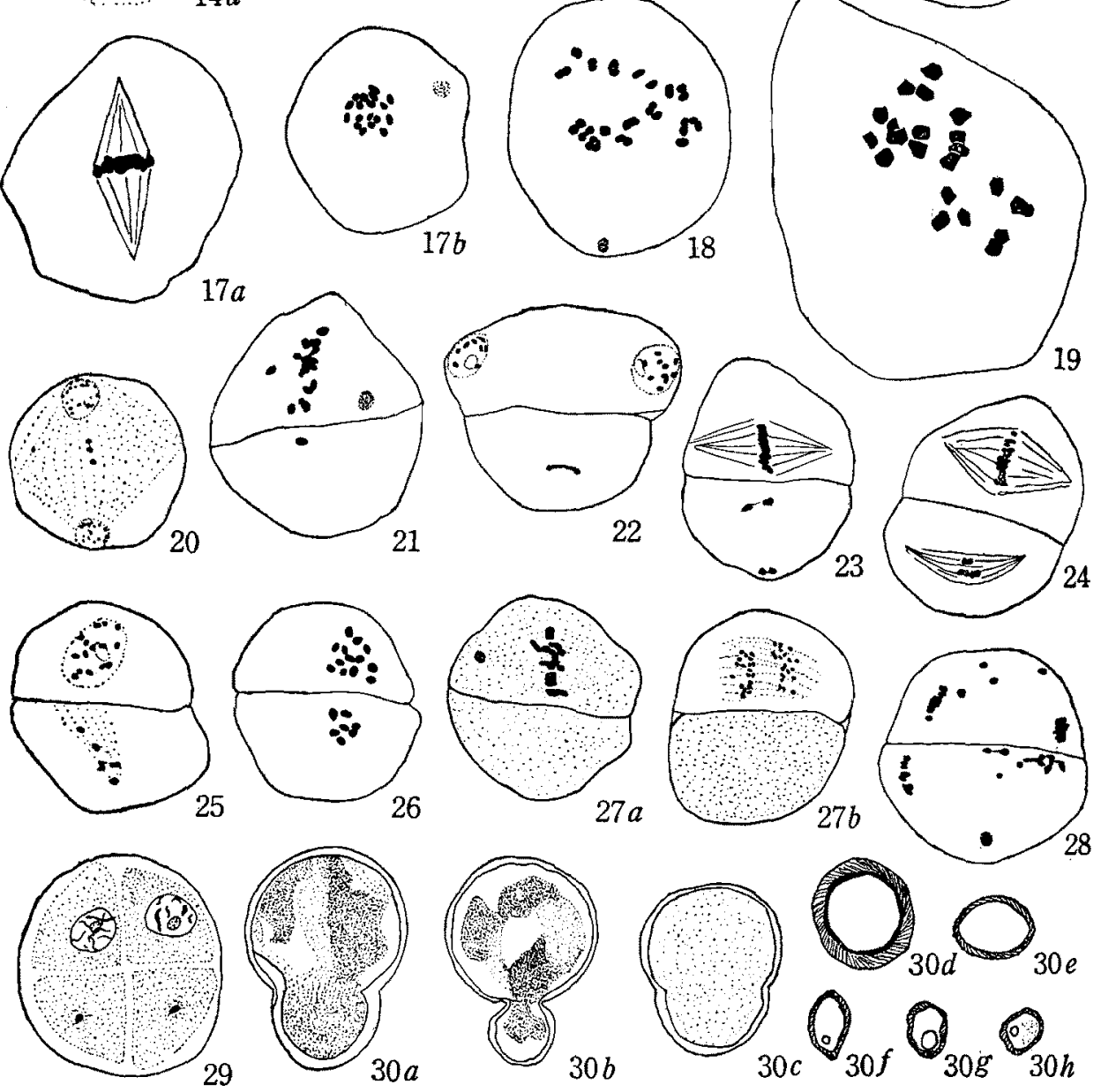

Figs. 14-30. Meiosis in Dsy-II. 14a, $\times$ ca $1760.14-17,19-28, \times 1150.18, \times 1350.29, \times 1050$. $30, \times$ ca 370 .

Table 2. Frequency of distribution of chromosomes

\begin{tabular}{c|ccccccccccc}
\hline \hline \multirow{2}{*}{ Plant } & $10 / 10$ & $12 / 8$ & $13 / 7$ & $14 / 6$ & $15 / 5$ & $16 / 4$ & $17 / 3$ & $18 / 2$ & $19 / 1$ & $20 / 0$ & Total \\
\hline Dsy-2 & 2 & 1 & 2 & 3 & 4 & 1 & 9 & 4 & 15 & 27 & 68 \\
\hline
\end{tabular}


chromosomes get included in the nuclei at the end of the anaphase-I. Telophase is thus clean but sometimes cells are found in which some laggards are still undergoing division at the equatorial plate (Fig. 20). Table 2 gives the frequency of distribution of chromosomes at the end of telophase-I.

The 19-1 distribution may be either due to 18 univalents moving to one pole and one bivalent disjoining or due to $19_{\mathrm{I}}$ moving to one pole and one to the other. Similarly the distributions in other cases may be owing to random distribution of univalents as also the occasional bivalent disjoining. The interkinesis is regular and cytokinesis is completed. The sizes of the daughter nuclei differ considerably depending on the number of chromosomes included. Figs. 24 to 27 show $17-3,15-5,13-7$ and 20-0 distribution at telophase-I; persistent nucleolus is also found.

Metaphase-II is regular, compact and a regular spindle is formed when the number of chromosomes is not too small. In cases where there are one or two chromosomes, no strong spindle is developed but a few threads are found (Fig. 21. 19-1 distribution at telophase-I; persistent nucleolus). The anaphase is regular where there are a number of chromosomes present and somewhat stretched out where only a few chromosomes are concerned. Irregular distribution occurs in the second division also and of ten laggards are found at the equator. However, in the majority of cases equational division is the rule and frequently precocious (Fig. 25). The single chromosome in a 19-1 division undergoes equational division rather delayed sometimes, while the other cell has already far advanced (Fig. 22) and of ten cytokinesis is completed. Fig. 23 is an anaphase-II showing 18-2 distribution, chromosomes undergoing equational division.

In cases where the distribution is $20-0$ the first cytokinesis is completed and in the second division the daughter cell which receives no chromosomes either shows no spindle development or sometimes faint or very rarely well formed spindle threads. The other cell behaves normally. Fig. 27a, shows anaphase-II with $\frac{20}{20} / 0$ distribution of chromosomes. The second division wall formation is completed irrespective of the number of chromosomes present

Table 3. Diameters of pollen grains in oc. micrometer divisions $(\times 1.58$ for $\mu)$

\begin{tabular}{c|ccccccccc}
\hline \multirow{2}{*}{ Plant } & $10-14$ & $15-19$ & $20-24$ & $25-29$ & $30-34$ & $35-39$ & $40-44$ & $45-49$ & Total \\
\hline $\begin{array}{c}\text { Dsy-1 } \\
\text { Sterile }\end{array}$ & 2 & 20 & 65 & 85 & 13 & 9 & 2 & 2 & 198 \\
Fertile & - & - & - & 2 & - & - & - & - & 2 \\
\hline $\begin{array}{c}\text { Dsy-2 } 2 \\
\text { Sterile }\end{array}$ & 17 & 84 & 59 & 3 & 2 & -1 & - & - & 165 \\
Fertile & - & - & 16 & 13 & 2 & 1 & 3 & - & 35 \\
\hline
\end{tabular}

resulting in four daughter cells (Fig. 29, tetrad formation with 19-1 distribution). Very often the resulting daughter cells are of unequal sizes. In 
cases where no chromosomes are preserit the wall formation may be slower and sometimes incomplete. The pollen is $70 \%$ sterile. Size and shape of pollen grains vary considerably (Fig. 30-a-h). The size frequencies of the pollen grains is given in Table 3 .

In some pollen mother cells at diakinesis it was found that two, sometimes three chromosomes were found separated from the others and forming an independent nucleus. These small nuclei had no nucleoli whereas the
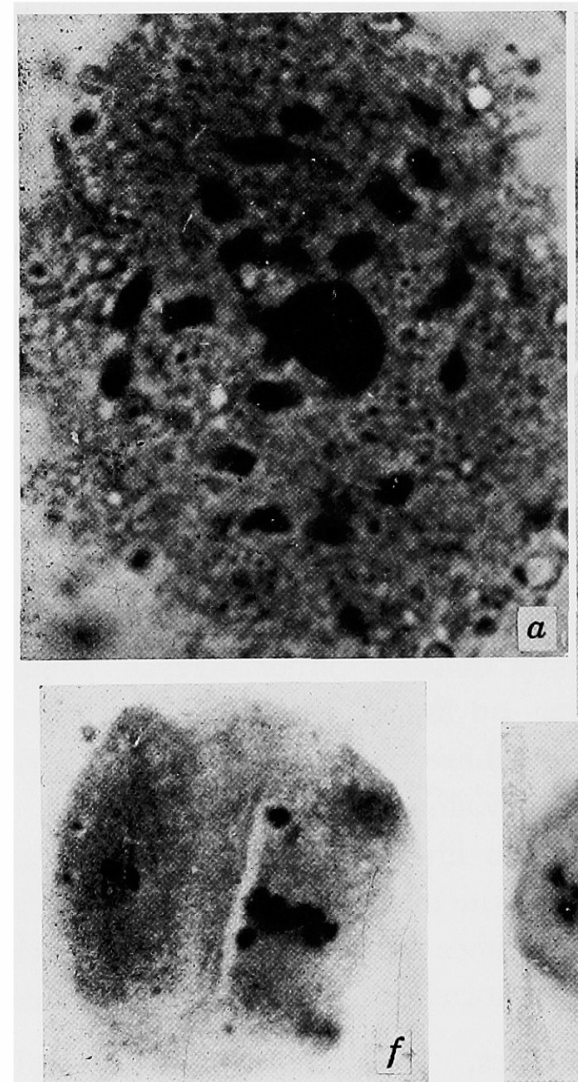

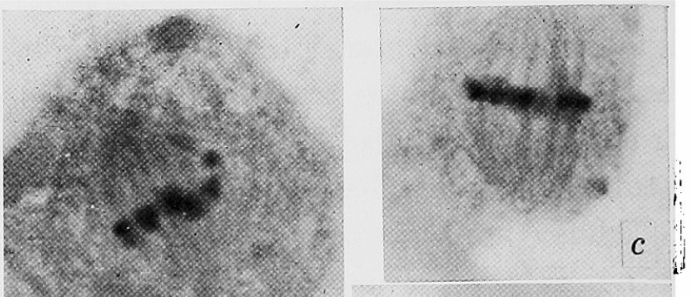

$b$

\section{$d$}

e

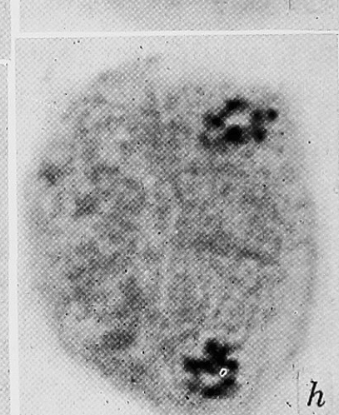

Fig. 31. All of meiosis in Dsy-2. a, diakinesis. $20_{\mathrm{I}} \times 1640$. b and $\mathrm{c}$, metaphase, with normal bipolar spindle and compact plate. $\times 570$. d, anaphase-I, $\times 570$. e, metaphase-II showing 19-1 distribution. $\times 785$. f, anaphase-II showing 2 in one daughter cell. $\times 950$. g, anaphase-II in a P.M.C. with $20-0$ distribution. $\times 785$. h, telophase-II. Cytokinesis in P.M.C. with 20-0 distribution. Cell plate in cell with chromosomes at telophase. $\times 785$.

larger ones had the usual nucleolus. The small cell undergoes nuclear changes along with the main one. The number of these abnormal cells being very small their further behaviour could not be followed. Similar abnormality has been noted in Tridax procumbens (Raghavan and Venkatasubban 1931) and in Tetrastigma sulcatum (Shetty 1956). Fig. 28 shows a P.M.C. with disturbed 2nd division; persistent nucleolus in one daughter cell.

The nucleolus is found persisting quite frequently both in the first and second divisions and becomes distributed to the daughter cells sometimes at 
random without division or it frequently divides haphazardly and the parts migrate to the poles and get included in the daughter cell. The most common position of the nucleolus in the P.M.C. is away from the spindle and more towards the wall (Figs. 15, 16, 17, 18, 21, 27, and 28).

The two types of asynaptic plants show a very high degree of sterility both male and female. In the first type the pollen tested with iodine showed very rarely two to three grains with contents while all the others were empty. Practically no seeds set even when dusted with pollen from fertile diploids. The second type on the other hand showed more fertile pollen grains and produced one to two and sometimes upto eight seeds per panicle, though this may be too small when compared to a normal one (Av. number set 3000). When dusted with pollen from a diploid normal plant the seed setting is sometimes better but when dusted with pollen from hybrid polyploid sorghums, or tetraploid maize and even such unrelated species as Pennisetum typhoides the number of seeds set is greater even approaching 25 to $30 \%$ of the normal fertile plants. This suggests a possible tendency to apomixis in this plant.

Inheritance. As mentioned above both types of desynapsis were met with in segregating progenies. The parents involved in these hybrids are all pure lines, self-fertile and the seed set is almost cent per cent. The meiosis of parents has shown complete synapsis of the homologous chromosomes. In the following is given the behaviour of the progenies.

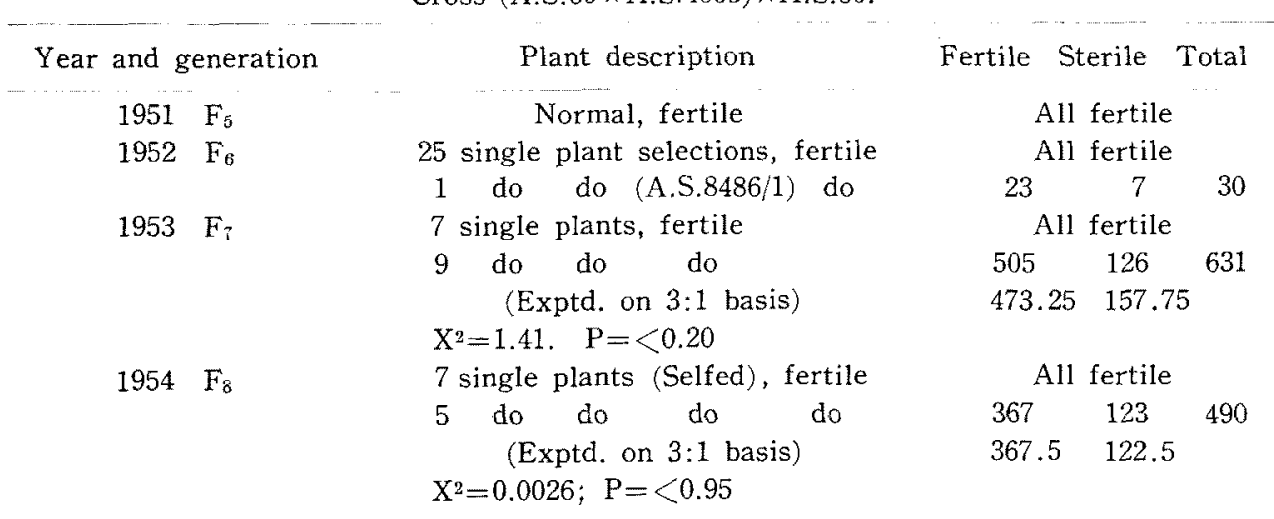

Family of Dsy-1. No seeds set in the sterile plants during 1952. The segregating progenies of 1953 were from open pollinated panicles, hence the discrepancy is higher while in 1954 all were selfed and the fit is close to a single factor segregation. Six seeds set in the sterile panicles during 1953 and only two germinated. These were found to behave as normal plants during 1954 . Meiosis was regular and full seed setting was observed. In 1954 again the panicles gave 10 shrivelled seeds which failed to germinate. The ratoons, however, gave six seeds which also failed to germinate. 
Family of Dsy-2 (Pure line A.S.681 Sen Cholam. Punjaipuliampatty). This variety of $S$. subglabrescens, red grained was hybridized with A.S. 4003 a strain of $S$. caudatum from Africa resistant to striga. The parent and the progeny were grown in trial plots. The progeny $\left(\mathrm{F}_{4}\right)$ is fertile and no sterile plants were observed. The parent lines were each from a single head thus representing a single plant progeny. One plot from such a single plant progeny of A.S.681 gave segregation for sterility (Fertile $=66$ : sterile $=17$. Total 83. Exptd. 62.25: 20.75. $\chi^{2}=0.89 ; \mathrm{P}=<0.70$ ).

The seed setting in this case was higher in the steriles than in the first type, the number varying from 1 to 8 in selfed panicles. Most of the seeds, however, are shrivelled. A few plants have been obtained from these selfed seeds.

\section{Discussion}

It is evident from the meiotic behaviour that these two types of desynapsis are different and they are simple Mendelian recessives to the normal condition.

Though in type-I there is a higher bivalent formation (6 per P.M.C.) the subsequent behaviour is such that the gametes are almost fully sterile. The ratoons have been continued to be grown for three years during which period there have been various changes in climate, temperature etc. Inspite of this at no time was even a single panicle found to have higher fertility. Seed setting was rare. Pollination from normal diploids did not alter the situation. It is possible that there may be gametic degeneration subsequent to meiosis. The rare seeds set showed in two cases that normal gametes with 10 chromosomes are occasionally formed. The resulting plants were completely fertile. Whether these were chance hybrids could not be said since the progeny from these two fertile plants was not raised.

In the second type on the other hand the pairing is very low, yet there is higher fertility of gametes as determined by pollen test. This has also been kept growing for over a year and the degree of fertility when selfed has remained very low. However, one or two seeds at least could be expected in a panicle.

Darlington (1937) has summarised the "causal relationships of different kinds of non-pairing" of which the first is the "Genotypic abnormality" (asynaptic mutants). This affects all chromosomes, there is a lack of precocity, reduced pachytene pairing to a variable extent resulting in insufficient numbers of chiasmata for regular metaphase pairing. Huskins and Hearne (1933) working on dwarf wheats and oats conclude that the lack of pairing is correlated with premature splitting of the chromosome threads and irregular contraction, of which the former is primary. Levan (1940) found strong and intimate pairing at pachytene but found at diplotene that no real chiasmata 
had been formed and this reduced precocity also produced a reduced contraction of the chromosomes at metaphase giving rise to the long "semimitotic" chromosomes. In the present materials though detailed pachytene study has not been made it is seen that in the first type the chromosomes at metaphase are slightly larger than in the second type, while in the latter there is complete contraction so as to render the chromosomes spherical. This may in part be due to shortness of the chromosomes compared those of Allium. Beadle and McClintock (1928) found in an asynaptic maize that the metaphase was highly irregular, univalents not always fully congressign, the spindle consisted of a major one in which the bivalents if any and some univalents were found and one to several minor spindles containing one or more univalents. This led to division of the microsporocytes into several unequal cells after M-II, each cell containing one or more nucle and each nucleus containing one or more chromosomes. This type of male sterility was observed to be accompanied by a certain amount of female sterility and several pollinations gave only sparsely filled ears. Beadle (19:0) described asynaptic maize and designated the gene as 'as'. At diakinesis, he reports 20-univalents and several spindles formed during M-I. One or more bivalents were frequently observed. Univalents were found scattered through several spindles and the spindle was much drawn out so that it became bent. Second division was nearly normal. Microsporocytes with 20 unsynapsed univalents in a late diakinesis-like stage with walls like that of spores and in later stages not distinguishable from the normal ones were met with. Such thickening of wall during meiosis was observed in the first type described here (Figs. 2c,3,6 and 8). Except for the solitary instance of a non-coalesced spindle in the type-I there have been no instances of multiple spindles. Powers and Dahl (1937) report again in maize asynapsis, a positive association between the degree of failure of pairing during diakinesis and metaphase- $\mathrm{I}$ and chromatin loss and this is affected by enviornment. Ramanujam and Parthasarathy (1935) observed in rice 24 univalents and irregular distribution at anaphase, spindles of different sizes, shapes and numbers, supernumerary and even split. Second division was generally normal. Microspores and microcytes of varying size and number were found. Krishnaswamy et al (1949) found in Pennisetum typhoides one to two bivalents formed in about $26 \%$ of P.M.Cs observed and this reduced considerably towards metaphase and anaphase. There is no full congression of univalents into a plate at metaphase $-\mathrm{I}$ and the spindle is often bent. Pal and Ramanujam (1940) described 24 univalents at diakinesis with occasional single bivalent and no metaphase plate formation, anaphase unequal and irregular distribution. Second division was more normal.

In the present materials the meiosis is remarkably regular especially in Dsy-II in which a very low proportion of bivalents are formed. The metaphase is normal with a well developed bipolar spindle. The anaphase 
is regular and no instance of chromatin brigdes were found. Laggards were rare. In the case of cells which receive fewer number of chromosomes, division follows as in the normal cells. This may probably be due to similar ' cooperative and balance effect' during the division of the pollen grains as reported by Barber (1941) in Uvularia. It is interesting to note that when dusted with pollen from unrelated plants like maize and pennisetum the seed set increased in the case of Dsy-II. However, it was highest when the sterile panicles were dusted with pollen from a $4 n$-sorghum hybrid plant. There is a possibility that in this plant seeds are produced to a certain extent apomictically as there are ample evidences to show that unreduced gamete formations are possible. Hadley and Mahan (1956) also mention the possibility of unreduced eggs in Sorghum vulgare. Huskins and Smith (1934) describe an asynaptic plant in Dakota Amber Sorgho and observe frequent multivalent formations. They consider this sort of pairing as only 'Homoeologous' rather than homologous segment pairing and that the asynaptic plant may have arisen as a mutant type. The asynaptic types reported here differ essentially from the above in giving only bivalents as the maximum association of chromosomes and not any multivalents. It may be that the chromosome complement of Dakota Amber Sorgho differs from that of the types discussed above. Since these occur in progenies which for generations gave no sterile plants, it can be concluded that these also have arisen as mutations and continued through heterozygous plants.

The two types described here have each behaved as simple Mendelian recessives to the normal condition. It has not been possible as yet to hybridize the two types of desynapsis to determine whether they are allelomorphic to one another or are independent genes. For the present it may be assumed that two separate genes are responsible for inducing these two types of desynapsis. Remarkable close parallel variation is observed between a number of mendelizing characters of maize and grain sorghums, so, to be in conformity with that of maize these two types of desynapsis may be designated as " as ${ }^{1}$ and as" ${ }^{2}$ ".

These plants have been utilized in hybridizing with other diploids as also tetraploids. A few plants from selfed panicles have also been obtained. The behaviour of these progenies is of much interest and will be reported elsewhere.

\section{Summary and conclusions}

1. Two types of desynapsis in the grain sorghums are reported. The desynapsis does not produce any variation in the morphological characters of the plants.

2. The meiosis is described in detail. In the first type upto six bivalents are found per P,M,C, while in the second it is 0.62 . The meiosis is without 
any aberrations and runs normal. The sterility is almost complete in the first type while it is about $70 \%$ in the second. Female sterility is accompanied with these male sterile types.

3. The inheritance of the two types is traced and they are each found to be simple Mendelian recessives. Gene symbols " $a s^{1}$ " and " as" " are proposed for them. It is assumed for the present that the two are independent of each other. These may be different from the asynapsis described by Huskins and Smith (l.c.). It is concluded that they should have arisen as mutations.

4. There is ample possibility for production of unreduced gametes especially in the second type and hence of apomixis also. A progeny of selfed panicles as also hybrids with tetraploids has been obtained and are under study. Their behaviour promises to be of much interest.

\section{Acknowledgements}

Our thanks are due to the Millets and Pulses Specialist for kindly permitting the use of the segregation data, and to Messrs. P. Chandrasekharan and D. Krishnaswamy for help in taking microphotographs.

\section{Literature cited}

Barber, H. N. 1941. Chromosome behaviour in Uvularia. J. Gent. 42: 223.

Beadle, G. W. 1930. "Genetical and cytological studies of mendelian asynapsis in Zea mays L." Cornell. Univ. Agric. Exp. Sta. Mem. 129.

- and McClintock, B. 1928. A genic disturbance of meiosis in Zea mays L. Science and dytological. 68: 433 .

Darlington, C. D. 1937. Recent advances in cytology. J and A Churchill Ltd. London.

Hadley, H. H. and Mahan, J. L. 1956. The cytogenetic behaviour of the progeny from a back cross (S. vulgare $\times S$. halepense $\times S$. vulgare). Agronomy J. 48 No. 3.

Huskins, C. L. and Hearne, E. M. 1933. Meiosis in asynaptic oats and wheat. J. Royal Micros. Soc. 53: 109. (Biol. Abstr. 1935, Entry 17605).

- and Smith, S. G. 1934. A cytological study of the genus Sorghum Pers. II. The meiotic chromosomes. J. Gent. 28: 387.

Krishnaswamy, N., Raman, V. S. and P. Madhava Menon, 1949. Abnormal meiosis in $F$. typhoides-I Desynapsis. Proc. Ind. Acad. Sci. 30, 195.

Levan, A. 1940. The cytology of Allium amplectans and occurrence in nature of its asynapsis. Hereditas 26: 353.

Li, H. W., Pao, W, K. and Li, C. H. 1945. Desynapsis in the common wheat. Amer. J. Bot. 32: 92.

Pal, B. P. and Ramanujam, S. 1940. Asynapsis in chilli (Capsicuum annum.). Curr. Sci. 9: 126.

Pao, W. K. and Li, H. W. 1948. Desynapsis and other abnormalities induced by high temperature. J. Gent. 48: 297.

Powers, Le Roy and Dahl, A. O. 1937. Failure of diakinesis and metaphase pairing and the behaviour during meiosis of univalent chromosomes in Zea mays L. J. Agric. Res. 54: 655.

Raghavan, T. S. and Venkatasubban, K. R. 1941. Contribution to the cytology of Tridax 
procumbens. Linn. Proc. Ind. Acad. Sci. 13B: 85-108.

Ramanujam, S. and Parthasarathy, N. 1935. Asynaptic mutant in Rice (Oryza sativa, L). Proc. Ind. Acad. Sci. 2B: 80.

Shetty, B. V. 1956. Cytogenetical studies in the cultivated grapes (Vitis vinifera L) of South India and related wild species. Madras University Thesis (unpub.). 